EPJ Web of Conferences 41, 04008 (2013)

DOI: $10.1051 /$ epjconf/20134104008

C Owned by the authors, published by EDP Sciences, 2013

\title{
Polarization-induced phase shift of ultrafast photocurrents
}

\author{
S. Priyadarshi, K. Pierz, and M. Bieler
}

Physikalisch-Technische Bundesanstalt, Bundesallee 100, D-38116 Braunschweig, Germany

\begin{abstract}
Shift and injection currents are known to occur for linearly and circularly polarized optical excitations of semiconductors, respectively. Here, we show with room-temperature experiments that for excitation of discrete transitions the frequency dynamics of the coherent polarization changes this phase rule significantly.
\end{abstract}

For above-bandgap optical excitation of semiconductors two main components of all-optically induced photocurrents have been recognized and are known as shift and injection currents [1]. These currents can be linked to a second-order optical nonlinear process associated with differencefrequency mixing. Microscopically, shift currents and injection currents result from a polar distribution of carriers in real and momentum space, respectively. They are also known as linear and circular photogalvanic currents, since they occur for optical excitation with linearly and circularly polarized light, respectively [1-3].

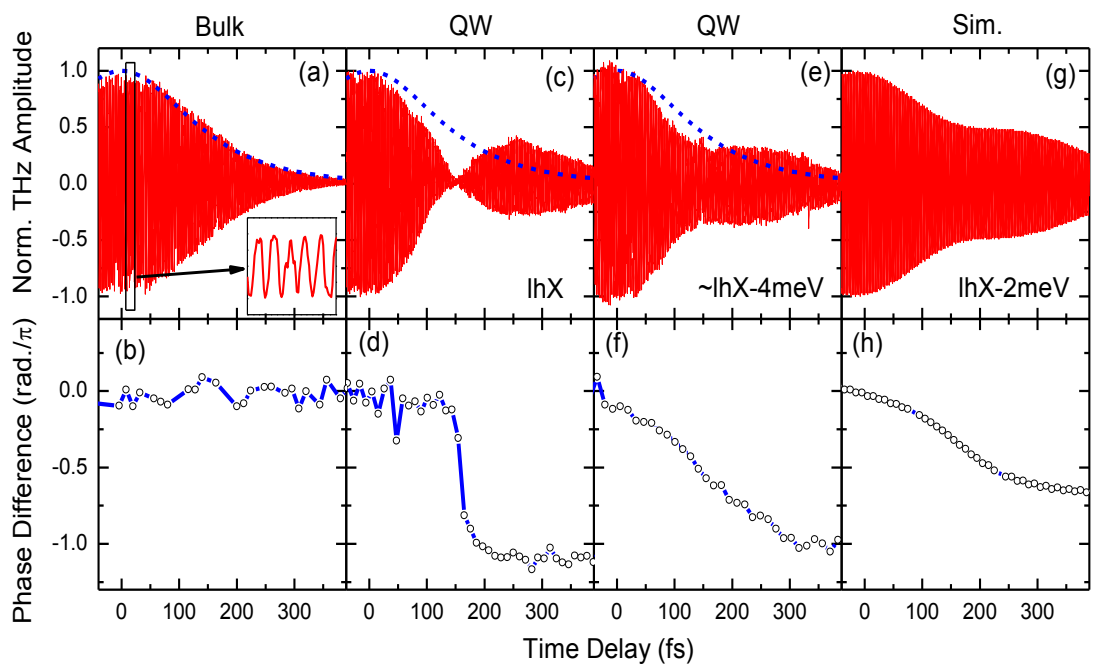

Fig. 1. THz amplitude versus time delay between the two excitation pulses in the (a) bulk sample for excitation of continuum transitions, (c) QW sample for excitation close to the lhX, (e) QW sample for the excitation energy detuned by $-4 \mathrm{meV}$ as compared to (c), and (g) simulation with a $-2 \mathrm{meV}$ detuning. The phase difference between the $\mathrm{THz}$ and optical interferograms are plotted underneath the corresponding THz interferograms in (b), (d), (f), and (h).

Here, we show that the frequency dynamics of the coherent polarization resulting from excitation of discrete transitions is capable of significantly altering this phase rule such that shift and injection currents occur for both, linear and circular polarizations. The measurements are performed on exciton transitions in a (110)-oriented, 5-nm wide GaAs quantum well (QW) sample, with the x, y, and $\mathrm{z}$ directions being parallel to the [001], [1 10], and [110] crystallographic directions, respectively. The sample is excited using an unchirped 150 -fs long orthogonally polarized pulse pair. The time

This is an Open Access article distributed under the terms of the Creative Commons Attribution License 2.0, which permits unrestricted use, distribution, and reproduction in any medium, provided the original work is properly cited. 
delay $(\tau)$ between the pulses having the same spectra can be precisely controlled using a piezo translation stage. The optical intensity of both pulses is only $\sim 3.5 \mathrm{MW} / \mathrm{cm}^{2}$ in order to reduce carriercarrier scattering, which contributes to dephasing of the coherent polarization. By controlling the time delay between the orthogonally polarized beams, shift and injection currents are generated along the y-direction [4]. The currents are detected by measuring the simultaneously emitted $\mathrm{THz}$ radiation in a typical electro-optic detection system [4]. All experiments are done at room temperature.

In Fig. 1a is plotted the THz amplitude versus time delay between the orthogonal polarizations measured in bulk GaAs, where injection currents are symmetry forbidden and only shift currents occur. The $\mathrm{THz}$ amplitude oscillates around zero. The envelope of the oscillations is similar to the envelope of an interferometric correlation signal resulting from the two excitation pulses (dashed line). In Fig. $1 \mathrm{~b}$ is plotted the difference between the $\mathrm{THz}$ and optical phases (extracted from the $\mathrm{THz}$ and optical interferograms, respectively) versus time delay. Both interferograms were measured simultaneously. To obtain an optical interferogram from an orthogonally polarized pulse pair a standard ellipsometric technique has been used. With this arrangement the optical interferogram can be used to specify the polarization state of the pulse pair: A phase difference between the optical and $\mathrm{THz}$ interferograms being equal to even (odd) multiples of $\pi / 2$ will be obtained if the $\mathrm{THz}$ amplitude is maximized for a phase delay between the two excitation pulses being equal to even (odd) multiples of $\pi / 2$. From Fig. $1 b$ it becomes apparent that the phase difference is (close to) zero for all time delays, showing that the maximum $\mathrm{THz}$ signal is obtained for linear polarization as expected in a scenario where only shift currents occur.

A similar measurement of the $\mathrm{THz}$ amplitude in the QW sample for optical excitation close to the light-hole exciton $(\mathrm{lhX})$ transition shows a different dependence on the time delay, see Fig. 1c. The envelope of the $\mathrm{THz}$ interferogram decays rather slower than the optical correlation (dashed line) which can be attributed to a longer dephasing time of the lhX. This longer dephasing even exists at room temperature in our (110)-oriented structures. In addition, a sharp dip in the $\mathrm{THz}$ interferogram at a time delay of $150 \mathrm{fs}$ occurs, see Fig. 1c. This dip results from a destructive interference between the shift currents generated in the bulk substrate and in the QW region [2]. The THz interferogram of the shift current from the bulk substrate dominates around $\tau=0$ and decays quite fast due to fast dephasing in the bulk substrate. In contrast, the shift current $\mathrm{THz}$ interferogram from the QW region decays slower due to slower dephasing in the QW and, hence, dominates for $\tau>150$ fs. At $\tau \sim 150$ fs both contributions cancel each other yielding a perfect dip in the $\mathrm{THz}$ interferogram. The counteraction of the two shift currents is also apparent in Fig. 1d where an abrupt phase jump of $\pi$ occurs at $\tau \sim 150$ fs.

The above behavior of the $\mathrm{THz}$ interferogram considerably changes if the excitation photon energy is slightly detuned from the $\mathrm{lhX}$, see Figs. 1e and $\mathrm{f}$. The dip in the $\mathrm{THz}$ interferogram nearly disappears and the phase varies gradually with a negative slope for negative detuning. For positive detuning a positive slope is obtained (not shown). Such gradual variation of phase cannot be explained with a superposition of shift and injection currents having a cosine and sine dependence on the phase delay, respectively, between the orthogonally polarized excitation fields. Rather it requires a phase shift in the cosine and sine terms as explained in the following.

Expressions for shift (SC) and injection currents (IC) can be obtained from the trace of the density matrix times an effective velocity matrix as described in [1]:

$$
\begin{gathered}
J_{I C}^{y}(t)=e \int \frac{d \mathbf{k}}{8 \pi^{3}} \sum_{n}\left[\rho_{n n}^{(2)} v_{n n}^{y}\right], \\
J_{S C}^{y}(t)=-\frac{e^{2}}{\hbar} \int \frac{d \mathbf{k}}{8 \pi^{3}} \sum_{m, n} \rho_{m n}^{(1)}\left[r_{n m ; y}^{x} E^{x}(t)+r_{n m ; y}^{y} E^{y}(t)\right] .
\end{gathered}
$$

Here, $\rho_{m n}^{(1)}$ and $\rho_{n n}^{(2)}$ are the first- and second-order solutions of the corresponding density matrix elements, $E^{x}(t)$ and $E^{y}(t)$ are the electric fields of the optical excitation, $\vec{r}_{n m ; y}$ is the generalized derivative of the dipole matrix element $\vec{r}_{n m}$ and $v_{n n}^{y}$ is the velocity matrix element along the y direction. From the above equations it is easy to show that the injection current results from an asymmetrically distributed population in momentum space while the shift current results from an asymmetric shift of carriers in real space. 
Solving the two-level Bloch equations for a detuned excitation up to second order in the electric field and inserting the resulting expressions into the equations above yields:

$$
\begin{aligned}
& \begin{array}{r}
\dot{J}_{I C}^{y}(t)+\frac{J_{I C}^{y}}{T_{S}}=\int \frac{d \mathbf{k}}{8 \pi^{3}} i \eta^{y x y}\left[\left\{f_{e n v, R}^{x}(t-\tau) E_{e n v}^{y}(t)+f_{e n v, R}^{y}(t) E_{e n v}^{x}(t-\tau)\right\} \sin \left(\omega_{c} \tau\right)\right. \\
\left.+\left\{f_{e n v, I}^{x}(t-\tau) E_{e n v}^{y}(t)-f_{e n v, I}^{y}(t) E_{e n v}^{x}(t-\tau)\right\} \cos \left(\omega_{c} \tau\right)\right]
\end{array}, \\
& \begin{array}{cc}
J_{S C}^{y}(t)=\int \frac{d \mathbf{k}}{8 \pi^{3}} \sigma_{s}^{y x y} & {\left[\left\{f_{e n v, R}^{x}(t-\tau) E_{e n v}^{y}(t)+f_{e n v, R}^{y}(t) E_{e n v}^{x}(t-\tau)\right\} \cos \left(\omega_{c} \tau\right)\right.} \\
\left.-\left\{f_{e n v, I}^{x}(t-\tau) E_{e n v}^{y}(t)-f_{e n v, I}^{y}(t) E_{e n v}^{x}(t-\tau)\right\} \sin \left(\omega_{c} \tau\right)\right]
\end{array} .
\end{aligned}
$$

Here, $\omega_{c}$ is the center frequency of the two optical excitation pulses and $T_{s}$ is the momentum scattering time which destroys the carrier asymmetry of the momentum-space distribution and leads to a decay of the injection current. The expressions $f_{e n v, R}^{x, y}(t)$ and $f_{e n v, I}^{x, y}(t)$ correspond to the real and imaginary parts, respectively, of the modified electric field envelope $f_{\text {env }}^{x, y}(t)=\int_{-\infty}^{t} E_{\text {env }}^{x, y}(x) \exp (-(\gamma+i \Delta)(t-x)) d x=E_{\text {env }}^{x, y}(t)(\gamma+i \Delta)^{-1}-\dot{E}_{\text {env }}^{x, y}(t)(\gamma+i \Delta)^{-2}+$ $\ddot{E}_{e n v}^{x, y}(t)(\gamma+i \Delta)^{-3}-\cdots$, where the modified electric field corresponds to the coherent polarization along a particular direction. Additionally, $\gamma$ is the dephasing rate, $\Delta$ is the detuning, and $\eta^{y x y}=$ $i \frac{4 e^{3}}{\hbar^{2}} \Delta_{12, \mathbf{k}}^{y} \operatorname{Im}\left(r_{12}^{x} r_{21}^{y}\right)=-\eta^{y y x}$ is a current tensor element for $J_{I C}^{y}(t)$ with $\Delta_{12, \mathbf{k}}^{y}=v_{11, \mathbf{k}}^{y}-v_{22, \mathbf{k}}^{y}$. As known from previous studies on injection currents, the tensor element is imaginary and antisymmetric in the last two Cartesian indices. The tensor element for $J_{S C}^{y}(t)$ is denoted by $\sigma_{s}^{y x y}=$ $\frac{2 e^{3}}{\hbar^{2}} \operatorname{Im}\left(r_{12}^{x} r_{21 ; y}^{y}-r_{21}^{y} r_{12 ; y}^{x}\right)=\sigma_{s}^{y y x}$ and is symmetric in the last two Cartesian indices.

In equation (3), the first term containing $\sin \left(\omega_{c} \tau\right)$ exists for all types of excitation while the term containing $\cos \left(\omega_{c} \tau\right)$ only appears for detuned excitation. Likewise an additional term with $\sin \left(\omega_{c} \tau\right)$ also appears in the shift current equation, see equation (4). Combining the $\sin \left(\omega_{c} \tau\right)$ and $\cos \left(\omega_{c} \tau\right)$ terms in equations (3) and (4), we can write $j_{I C}^{y}(t)+\frac{J_{I C}^{y}}{T_{S}} \propto \sin \left(\omega_{c} \tau+\alpha(t, \tau)\right)$ and $J_{S C}^{y}(t) \propto$ $\cos \left(\omega_{c} \tau+\alpha(t, \tau)\right)$. Here $\alpha(t, \tau)$ is a time-delay dependent phase factor which results in a gradually varying phase difference as experimentally obtained in Fig. 1e and f. Our arguments are well supported by simulations for detuned excitation of a two-level system [5], see Fig. $1 \mathrm{~g}$ and h, which agree very well with the experiments.

Microscopically, the time-delay dependent phase term $\alpha(t, \tau)$ results from the non-instantaneous frequency dynamics of the coherent polarization, whose frequency approaches the resonance frequency of the two-level system after the driving field given by the optical excitation is gone. Although such non-instantaneous frequency dynamics of the coherent polarization has already been observed in four-wave mixing experiments [6], its influence on ultrafast all-optically generated currents has not been explored so far. In fact, the above analysis shows that polar distributions of carriers in real and momentum spaces, being at the origin of shift and injection currents, can also be obtained with other than linear and circular excitations, respectively.

In conclusion, we have shown that for a detuned excitation of discrete transitions shift and injection currents experience a phase shift. Such deviations result from the nonlinear frequency dynamics of the coherent polarization, which, in this particular case, is obtained using detuned excitation of excitons in a GaAs QW sample.

\section{References}

[1] J. E. Sipe and A. I. Shkrebtii, Phys. Rev. B 61 (2000) 5337.

[2] M. Bieler, K. Pierz, U. Siegner, and P. Dawson, Phys. Rev. B 76 (2007) 161304(R)

[3] S. Priyadarshi, A. M. Racu, K. Pierz, U. Siegner, M. Bieler, H. T. Duc, J. Förstner, and T. Meier, Phys. Rev. Lett. 104 (2010) 217401.

[4] M. Bieler, K. Pierz, and U. Siegner, J. Appl. Phys. 100 (2006) 83710.

[5] We obtain the best agreement between experiment and simulation for slightly smaller detuning in the simulation. We attribute this effect to inhomogeneous broadening of the excitons in our QW sample, which is not accounted for in the simulations.

[6] J. Shah, Ultrafast spectroscopy of semiconductors and semiconductor nanostructures, Springer, Berlin, 1999. 